\title{
Validación y puntos de corte de la versión en español del Cuestionario de Edades y Etapas: Social-emocional, 2da edición (ASQ:SE-2), en un entorno ambulatorio urbano de Lima-Perú.
}

Validation and cut-off values of the Spanish version of Ages \& Stages Questionnaires: Social-Emotional $2^{\text {nd }}$ edition (ASQ:SE-2) in an urban outpatient setting at Lima-Perú.

\author{
Adriel Gudiel-Hermoza 1,a, Jorge Gudiel-Hermoza ${ }^{1, a}$, Yuri Mancilla-Gudiel 1,b, Daniel Guillen-Pinto ${ }^{2, c}$
}

\section{RESUMEN}

Objetivos: Determinar la confiabilidad, validez y puntos de corte de la versión en español del Cuestionario de Edades y Etapas, 2 da edición (del inglés Ages \& Stages Questionnaires: Social-Emotional, 2nd Edition (ASQ: SE2). Material y Métodos: Mediante muestreo por conveniencia se aplicaron formas del cuestionario ASQ: SE-2 a padres de niños entre 15 y 48 meses de edad. La confiabilidad se determinó por medio de la consistencia interna del coeficiente alfa ordinal $(\alpha)$ y la validez de contenido mediante la correlación ítem-test (r). Los puntos de corte para establecer zonas de riesgo se determinaron empleando el percentil 65 y la curva ROC. Resultados: De un total de 236 cuestionarios aplicados, el coeficiente alfa ordinal se ubicó entre aceptable y bueno $(\alpha=0.73-0.87)$ para los 5 cuestionarios de intervalos de edad entre 18 y 48 meses. El índice de correlación de prueba de ítems mostró un buen poder discriminatorio para la mayoría de los ítems ( $r>0.30)$, con la excepción de un ítem para los meses 30, 36 y 48. Nuevos puntos de corte para los intervalos de edad entre 15 y 48 meses fueron determinados. Conclusiones: La versión en español de ASQ: SE-2 aplicada a padres de niños de 15 a 48 meses de edad en un entorno ambulatorio y en un área urbana de Lima-Perú, demostró ser una herramienta confiable y válida para la vigilancia y detección de etapas del desarrollo emocional.

PALABRAS CLAVE: Desarrollo social-emocional, ASQ:SE-2, Confiabilidad, validez.

Clínica Mundo Salud. Lima, Perú.

Universidad Peruana Cayetano Heredia. Lima, Perú.

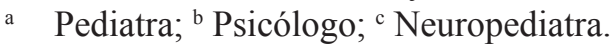




\section{SUMMARY}

Objectives: A study was carried out to determine the reliability, validity and cut-off points of the Spanish version of Ages \& Stages Questionnaires: Social-Emotional, $2^{\text {nd }}$ Edition (ASQ:SE-2). Material and Methods: Questionnaire forms of ASQ:SE-2 between 15 to 48 months were applied to parents of children chosen by convenience sampling. Reliability was determined by the internal consistency through Ordinal alpha coefficient ( $\alpha$ ), and validity of content through Item-test correlation (r). The cut-off points to establish risk zones were determined using the 65 th percentile and ROC curve. Results: Two hundred thirty-six questionnaires were applied. The Ordinal alpha coefficient reached a between acceptable to good level ( $\alpha=0.73-0.87)$ for all 5 age-intervals questionnaires between 18 to 48 months. The Item-test correlation index pointed a good discriminatory power for most items $(r>0.30)$, with the exception of one item for the months 30, 36 and 48. New cut-off points for the intervals-age between 15 - 48 months were determined. Conclusions: The Spanish version of ASQ: SE-2, applied to parents of children from 15 to 48 months of age in an outpatient setting from an urban area of Lima-Peru proved to be a reliable and valid tool for surveillance and screening of steps of social-emotional development.

KEYWORDS: Social-emotional development, ASQ-SE-2, validity, reliability, screening.

\section{INTRODUCCIÓN}

El desarrollo socioemocional en la infancia ocurre a la par del desarrollo psicomotor en general e involucra el establecimiento del vínculo afectivo entre padres e hijos, capacidad de autorregulación del niño, autonomía, adaptación, interacción y comunicación social (1). Las alteraciones del desarrollo socio emocional ocurren en alrededor del 15\% de la población infantil y pueden ser objeto de vigilancia, detección y evaluación $(2,3)$. El uso de pruebas de detección es aún limitado en la práctica ambulatoria pediátrica y la sensibilidad del clínico para detectar alteraciones leves suele ser limitado, por lo que se requiere el uso de instrumentos validados para aplicación local (4-6). El Cuestionario de Edades y Etapas: Socio-emocional, 2da edición (ASQ:SE-2), fue desarrollado el 2009 en EE. UU. (7). Es un instrumento de vigilancia del desarrollo socioemocional y de detección de sus alteraciones. Es considerado un complemento del Cuestionario de edades y etapas tercera edición (ASQ$3)$, cuestionario que explora el desarrollo psicomotor general del niño $(8,9)$. El Comité de la infancia de la Academia americana de pediatría (AAP) y "Bright futures", recomiendan la vigilancia del desarrollo psicomotor, socioemocional y sus alteraciones de manera estructurada y sistematizada con el uso de herramientas estandarizadas y validadas a los 9, 18 y 24 ó 30 meses de edad $(2,10)$. Con la finalidad de hacer uso de la versión en español del ASQ:SE-2 nos preguntamos si es un instrumento confiable y válido. Además de determinar puntos de corte para los 5 cuestionarios comprendidos entre los 15 meses 0 días a 53 meses 30 días en un entorno de consulta externa población urbana de la ciudad de Lima.

Se desarrolló el presente estudio de validación con la finalidad de determinar la confiabilidad, validez de contenido y puntos de corte de la versión en español del ASQ:SE-2 en un entorno de consulta externa en una zona urbana de la ciudad de Lima-Perú.

\section{MATERIAL Y MÉTODOS}

Población y lugar de estudio: La población objetivo fueron padres de niños con edades entre los 15 meses 0 días a 53 meses 30 días, mediante muestreo por conveniencia. El lugar de estudio fue la consulta ambulatoria del servicio de pediatría de una Clínica particular ubicado en la zona norte de Lima con afluencia de pacientes de los distritos de Los Olivos, San Martin de Porres, Independencia y Comas; zonas consideradas de nivel socioeconómico medio. El estudio se llevó a cabo en el periodo de agosto 2017 a febrero 2019.

Criterios de inclusión y exclusión: Se incluyó en el estudio a los niños que fueron atendidos por control de niño sano, inmunizaciones y afecciones pediátricas de la consulta cotidiana (respiratorias, gastrointestinales y dérmicas, leves) . Se excluyo del estudio los cuestionarios que no pudieron ser completados por los padres por acudir con sus niños con enfermedad moderado o severa o requerir atención por el servicio de emergencia u hospitalización); además, si los niños tuvieran malformaciones congénitas múltiples, estar 
Validación y puntos de corte de la versión en español del Cuestionario de Edades y Etapas: Social-emocional, 2da edición.

en terapia anticonvulsiva, quimioterapia, hormonal o de radiación.

Instrumento: La versión en español del Cuestionario de Edades y Etapas: Social - emocional, 2 da edición (ASQ-SE-2) (7), en su versión completa está conformado por 9 cuestionarios con intervalos de edad de 6 meses desde 1 mes a 72 meses. Son cuestionarios de un solo factor que evalúa el desarrollo social-emocional en sus aspectos de autorregulación, cumplimiento, funcionamiento adaptativo, autonomía, afecto, comunicación social e interacción con las personas. Son cuestionarios completados por los padres en general en 10 a 15 minutos cada uno. El número de ítems por cuestionario varió de 31 a 36. Para su puntuación cada ítem tuvo 3 opciones excluyentes: "Frecuente o siempre", "A veces", "Rara vez o nunca" y una cuarta no excluyente: "Preocupante". Las opciones excluyentes tuvieron asignada una letra que representó un puntaje $(\mathrm{Z}=0, \mathrm{~V}=5, \mathrm{X}=10)$ y si marcó la opción no excluyente se le asignó 5 puntos. Según la puntuación obtenida podría caer en una de 3 zonas: "Dentro de las expectativas", "Observar" o monitoreo y "Consultar" o de riesgo.

La consistencia interna medida por el coeficiente alfa fue alta, entre $0,71-0,90$ y un alfa total de 0,84 . Confiabilidad Test-retest: 0,89, sensibilidad general: 0,81, especificidad general: 0,84 (7). En el 2015, el $A S Q-S E$ fue modificado, habiéndose incluido 9 preguntas a 5 cuestionarios que van de 18 meses a 48 meses para ayudar a identificar a niños con posible riesgo de autismo (11). Para el presente estudio se eligió la validación de estos 5 cuestionarios.

Definición de controles y casos: El diagnóstico clínico de casos y controles estuvo a cargo del equipo de pediatría del desarrollo conformado por 1 pediatra, 2 psicólogos clínicos y 2 terapistas de lenguaje. Los "controles" o "sanos" fueron definidos como niños cuyos puntajes totales alcanzados se situaron en el área "dentro de las expectativas" y además el criterio clínico del pediatra corroboró su situación. La condición de "caso" fue definido cuando el puntaje total alcanzado en el cuestionario se situó en zona de "monitoreo" o "muy por debajo" y además tener algún trastorno del neurodesarrollo. Se empleó como prueba de oro el Criterio clínico para diagnóstico de casos, basado en la identificación de casos y codificación según el CIE-10 para trastornos del neurodesarrollo. Los diagnósticos de los niños considerados "casos" no se incluyeron en el presente informe.
Procedimiento: El procedimiento de aplicación del $A S Q-S E-2$ fue establecido como parte del proceso de vigilancia del desarrollo socioemocional de niños entre los 15 meses 0 días a 53 meses 30 días ( 5 cuestionarios). Los cuestionarios fueron completados por los padres en el área de espera o triaje previo al ingreso a la consulta, confiriéndose un tiempo de llenado de 10 a 15 minutos, para ello se dieron instrucciones de un correcto llenado, proporcionándose lápiz, papel y el cuestionario correspondiente a la edad del niño; en este proceso el personal de enfermería verificó el adecuado llenado de los cuestionarios y respondiendo a las inquietudes de los padres sobre ello. Se procedió luego a asignar los puntajes al cuestionario y ésta se adjuntó a la historia clínica del niño para su revisión por el pediatra. Ya en consulta, el pediatra estableció el puntaje alcanzado y determinar si el niño se encontraba o no en zona de riesgo. De hallarse en zona de riesgo se procedió a comunicar al padre de ello y recomendar que el niño deba ser sometido a mayor evaluación con la finalidad de llegar a establecer o no un diagnóstico clínico acompañado de alteración socioemocional.

Análisis Estadístico: Se determinó la confiabilidad de la prueba por análisis de consistencia interna mediante la aplicación de correlaciones tetracóricas entre sus ítems, obteniéndose el Coeficiente alfa ordinal $(\alpha)(12)$. Se consideró que el cuestionario tenía consistencia interna adecuada si el $\alpha>0,70$. La validez de contenido, determinando la validez de ítems mediante correlaciones policóricas (PCC) con la técnica Ítem-test (rho) en la cual la distribución de cada ítem se correlacionó con la distribución del puntaje total por cuestionario. Un ítem fue considerado válido si su rho $>0,30$. Siendo el tamaño muestral obtenido por conveniencia, se calculó el poder estadístico del estudio estimando el coeficiente de correlación lineal para la validez de los ítems. $Z_{1-\beta}=\sqrt{n-3} \frac{1}{2} \ln \left(\frac{1+r}{1-r}\right)-Z_{1-\alpha / 2}$. Al 95\% de confianza el valor de $Z_{1-\alpha / 2}$ fue 1.96; el valor de $r$ se espera fuera mayor o igual a 0.300 para que el ítem fuera válido y el poder estadístico sería $1-\beta$ que se obtuvo con la función de distribución normal tomando como argumento a $Z_{1-\beta}$.

Para cada una de las pruebas, el poder estadístico fue:

\begin{tabular}{lrrr}
\hline ASQ:SE-2 & $\mathbf{n}$ & $\mathbf{Z}_{\mathbf{1}-\boldsymbol{\beta}}$ & Potencia \\
\hline $18 \mathrm{~m}$ & 58 & 0.335 & $63 \%$ \\
$24 \mathrm{~m}$ & 57 & 0.315 & $62 \%$ \\
$30 \mathrm{~m}$ & 35 & -0.209 & $42 \%$ \\
$36 \mathrm{~m}$ & 60 & 0.377 & $65 \%$ \\
$48 \mathrm{~m}$ & 26 & -0.476 & $32 \%$ \\
\hline
\end{tabular}


El poder estadístico o potencia de cada cuestionario fue adecuado excepto para los cuestionarios de $30 \mathrm{y}$ 48 meses que tuvieron poco poder estadístico para detectar la validez de un ítem posiblemente por tener un tamaño muestral pequeño.

La determinación de los puntos de corte para cada uno de los 5 cuestionarios siguió la misma metodología empleada en el manual del usuario del ASQ:SE-2 $(7,13)$. Previo a ello se determinó el número de niños considerados controles $(n=197)$ y casos $(n=39)$, según las definiciones antes mencionadas. Así, se obtuvo el primer punto de corte para la zona de "Observar", considerando solo a los controles y tomando el percentil 65 de sus puntajes directos. El segundo punto de corte se obtuvo a través de una curva ROC donde el índice de Youden indicó la máxima sensibilidad y la máxima especificidad. Este índice equivalió a un puntaje estandarizado y se transformó a un puntaje directo obteniéndose el punto de corte para la zona de riesgo o "Consultar".

Estos puntos de corte demarcaron 3 zonas: La primera, "Dentro de las expectativas", comprendido en el área previo al primer punto de corte. Una segunda área de "Observar" o monitoreo, comprendido entre el primer y segundo punto de corte, y una tercera área de "Consultar" o de riesgo, ubicado por encima del segundo punto de corte. El análisis general para el proceso de validación se desarrolló con el uso del programa STATA 14.

El estudio fue revisado y aprobado previa a su ejecución por el Comité de ética del establecimiento y el consentimiento de los padres para completar los cuestionarios se obtuvo de manera verbal.

\section{RESULTADOS}

Se analizaron 236 cuestionarios del ASQ:SE-2, correspondiendo a 229 niños cuyos intervalos de edad de los cuestionarios estuvieron entre 18-48 meses. De ellos 137 (58\%) correspondieron al sexo masculino y 99 (42\%) sexo femenino. Los cuestionarios considerados controles fueron 197 (83,5\%), mientras que los casos $39(16,5 \%)$. No hubo diferencia estadísticamente significativa entre el grupo de hombres y mujeres con respecto a la condición de casos $(\mathrm{p}=0,155)$; es decir, los casos pudieron presentarse tanto en hombres como en mujeres con la misma probabilidad. Según la Tabla 1, la distribución por intervalos de edad y condición de controles o casos, estuvieron distribuidos de manera casi homogénea. Tampoco hubo diferencia
Tabla 1. Distribución según intervalos de edad del cuestionario ASQ:SE-2 y condición de caso o control *

\begin{tabular}{lcccc}
\hline & \multicolumn{2}{c}{ Control } & \multicolumn{2}{c}{ Caso } \\
Edad (m) & n & \% & n & \% \\
\hline 18 & 45 & 22,8 & 13 & 33,3 \\
24 & 47 & 23,9 & 10 & 25,6 \\
30 & 30 & 15,2 & 5 & 12,8 \\
36 & 52 & 26,4 & 8 & 20,5 \\
48 & 23 & 11,7 & 3 & 7,7 \\
Total & 197 & 100,0 & 39 & 100,0 \\
\hline
\end{tabular}

$\mathrm{m}=$ meses. $(*)$ : No hubo diferencias según intervalo de edad $y$ condición de caso o control $(\mathrm{p}=0,638)$.

Tabla 2. Consistencia Interna del $A S Q-S E-2$ según el Coeficiente alfa ordinal por cuestionario

\begin{tabular}{llc}
\hline Mes & n & Alfa ordinal \\
\hline 18 & 58 & 0,781 \\
24 & 57 & 0,824 \\
30 & 35 & 0,772 \\
36 & 60 & 0,872 \\
48 & 26 & 0,733 \\
\hline
\end{tabular}

Un $\alpha>0,70$ es considerado aceptable.

estadísticamente significativa entre ambos grupos por edad $(\mathrm{p}=0,638)$.

Consistencia interna: Se determinó la consistencia interna como una medida de confiabilidad del ASQ:SE-2 mediante el Coeficiente alfa ordinal, ésta alcanzó valores $>0,73$ para cada uno de los 5 cuestionarios; considerados de aceptable a bueno (tabla 2).

Validez de Ítems: Como se muestra en la tabla 3, los valores de la correlación ítem-test (r) para los 5 cuestionarios fueron altos $(r>0,30)$, indicando un buen poder discriminatorio por ítem. Sin embargo, para los cuestionarios de 30, 36 y 48 meses, hubo sólo 1 ítem que no resultó válido (ítems 32,6 y 5 respectivamente). Puntos de corte: En la tabla 4, se muestran los valores hallados de los 2 puntos de corte para cada uno de los 5 cuestionarios del ASQ:SE-2 con los que se establecen 3 áreas ("dentro de las expectativas", "monitoreo" y "riesgo"). En la misma tabla 4 para efectos comparativos se adjuntan los valores de primer y segundo puntos de corte según lo hallado en el presente estudio y los indicados en el Manual del $A S Q: S E-2$ original. 
Validación y puntos de corte de la versión en español del Cuestionario de Edades y Etapas: Social-emocional, 2da edición.

Tabla 3. Validez de ítems según cuestionario por edad (ASQ:SE-2) ( $\mathrm{n}=236)$

\begin{tabular}{cccccc}
\hline & mes $\mathbf{1 8}$ & mes 24 & mes 30 & mes 36 & mes $\mathbf{4 8}$ \\
Ítem & rho & rho & rho & rho & rho \\
\hline 1 & 0,479 & 0,652 & 0,630 & 0,646 & 0,463 \\
2 & 0,335 & 0,638 & 0,638 & 0,547 & 0,620 \\
3 & 0,824 & 0,327 & 0,612 & 0,313 & 0,941 \\
4 & 0,374 & 0,508 & 0,625 & 0,320 & 0,371 \\
5 & 0,353 & 0,608 & 0,767 & 0,463 & 0,023 \\
6 & 0,450 & 0,481 & 0,698 & 0,005 & 0,433 \\
7 & 0,349 & 0,364 & 0,317 & 0,491 & 0,314 \\
8 & 0,323 & 0,555 & 0,305 & 0,389 & 0,649 \\
9 & 0,320 & 0,328 & 0,592 & 0,479 & 0,469 \\
10 & 0,446 & 0,504 & 0,371 & 0,406 & 0,613 \\
11 & 0,349 & 0,520 & 0,824 & 0,644 & 0,318 \\
12 & 0,424 & 0,521 & 0,697 & 0,340 & 0,314 \\
13 & 0,539 & 0,375 & 0,821 & 0,429 & 0,453 \\
14 & 0,534 & 0,331 & 0,429 & 0,327 & 0,469 \\
15 & 0,556 & 0,670 & 0,593 & 0,457 & 0,303 \\
16 & 0,300 & 0,431 & 0,554 & 0,446 & 0,361 \\
17 & 0,351 & 0,503 & 0,321 & 0,398 & 0,689 \\
18 & 0,428 & 0,580 & 0,638 & 0,585 & 0,614 \\
19 & 0,657 & 0,538 & 0,747 & 0,361 & 0,781 \\
20 & 0,465 & 0,341 & 0,665 & 0,329 & 0,442 \\
21 & 0,352 & 0,384 & 0,543 & 0,591 & 0,405 \\
22 & 0,399 & 0,412 & 0,645 & 0,386 & 0,514 \\
23 & 0,394 & 0,500 & 0,789 & 0,564 & 0,433 \\
24 & 0,424 & 0,419 & 0,450 & 0,422 & 0,735 \\
25 & 0,301 & 0,398 & 0,336 & 0,624 & 0,622 \\
26 & 0,467 & 0,653 & 0,644 & 0,577 & 0,302 \\
27 & 0,596 & 0,645 & 0,378 & 0,497 & 0,852 \\
28 & 0,347 & 0,392 & 0,482 & 0,539 & 0,350 \\
29 & 0,343 & 0,611 & 0,532 & 0,366 & 0,568 \\
30 & 0,386 & 0,530 & 0,810 & 0,453 & 0,532 \\
31 & 0,372 & 0,525 & 0,311 & 0,376 & 0,405 \\
32 & & & $-0,069$ & 0,457 & 0,687 \\
33 & & & 0,354 & 0,431 & 0,402 \\
34 & & & & 0,341 & 0,466 \\
35 & & & & 0,388 & 0,771 \\
36 & & & & & 0,834 \\
\hline & & & & & \\
\hline
\end{tabular}

\section{DISCUSIÓN}

Un adecuado desarrollo social y emocional del niño indica un funcionamiento adaptativo del cerebro derecho con bases en el apego y regulación emocional alcanzados en edades tempranas (1). Vigilar e identificar las alteraciones del desarrollo socioemocional del niño requiere el uso de instrumentos estandarizados como el ASQ:SE-2 (7), lo cual requiere esté debidamente validado y con puntos de corte locales. 
Tabla 4. Comparativos de puntos de corte: $A S Q: S E-2$ (Según percentil 65 y Curva ROC)

Primer y segundo puntos de corte

\begin{tabular}{lcccc}
\hline & \multicolumn{2}{c}{$\begin{array}{c}\text { Según muestra } \\
\text { del estudio }\end{array}$} & \multicolumn{2}{c}{$\begin{array}{c}\text { Según muestra } \\
\text { del Manual (7) }\end{array}$} \\
Mes & 1er. & 2do. & 1er. & 2do. \\
\hline 18 & 52 & 57 & 50 & 65 \\
24 & 56 & 72 & 50 & 65 \\
30 & 59 & 67 & 65 & 85 \\
36 & 69 & 81 & 75 & 105 \\
48 & 56 & 81 & 70 & 85 \\
\hline
\end{tabular}

1er: Indica primer punto de corte según percentil 65.

2do: Indica segundo punto de corte mediante Curva ROC.

En su primera versión en el idioma inglés (ASQ:SE), de una muestra total de 2633 niños, se aplicó la prueba a 153 niños hispanohablantes, habiendo requerido para ello un grupo de expertos para la traducción al idioma español (14). Para su segunda edición, la validación del ASQ:SE-2 se basó en una población encuestada de 16424 cuestionarios (14 074 niños) donde, un 7,3\% (1 032 cuestionarios) correspondieron a niños de familias hispanas o latinas. Sin embargo, no se llegó a determinar la validez y confiabilidad de la prueba para esa población específica (7).

Para el presente estudio no llegamos a realizar una adaptación cultural y lingüística previa. Sin embargo, en el transcurso del estudio se pudo percibir por parte de los aplicadores y los padres que la versión traducida al español de los cuestionarios fue de fácil lectura, comprensible y breve.

El estudio de Dolata J., 2015 (11) fue el pionero en establecer la utilidad del ASQ:SE-2 como un instrumento de detección de niños con riesgo de autismo. Para la segunda edición del ASQ:SE-2 se había agregado 9 preguntas a cada uno de los 5 cuestionarios comprendidos entre 18 y 48 meses con la finalidad específica de aumentar la sensibilidad de detección del autismo $(7,11)$.

En el presente estudio, la consistencia interna mediante el coeficiente alfa ordinal alcanzó valores mayores al 0,73 , considerados de aceptables a buenos en cada uno de los 5 cuestionarios de análisis; siendo concordantes con lo referido en el Manual del ASQSE-2, donde el $\alpha=0,82(0,67-0,91)$.

Heo et al., (15), en un estudio de adaptación intercultural Coreana de la primera edición del
ASQ: SE, además de hallar una confiabilidad testretest de 0.84 de los cuestionarios completados por los padres en tiempos sucesivos, hallaron un coeficiente alfa aceptable, $\alpha=0,68(0,56-0,77)$. En lo correspondiente a validez de contenido, según nuestros hallazgos, mediante la correlación ítem-test para los 5 cuestionarios fue alta, indicando buen poder discriminatorio por ítem.

En general los puntos de corte del ASQ:SE-2 original y los determinados en el presente estudio para los 5 cuestionarios validados, no difirieron de manera importante. En estudios con mayor tamaño poblacional estos puntos de corte representarán con mayor precisión a la población estudiada. De manera general existen muy escasos estudios de validación de este instrumento a contextos clínicos ambulatorios en idioma español en general y para la región de Latinoamérica y el Perú en particular.

El proceso de validación de una prueba considera varias formas de establecer si es confiable y valida. Tal como se indicó en la sección de métodos, el poder estadístico o potencia de cada cuestionario fue adecuado excepto para los cuestionarios de $30 \mathrm{y}$ 48 meses que tuvieron poco poder estadístico para detectar la validez de un ítem debido a un tamaño muestral pequeño.

No obstante ello, con las medidas realizadas, se podría establecer que para efectos de iniciar un proceso de vigilancia y tamizaje del desarrollo socio emocional en niños entre 15 meses 0 días a 53 meses 30 días, estos cuestionarios fueron válidos y confiables para la población estudiada.

Es importante considerar que el presente estudio validó los 5 cuestionarios que tienen incluidas preguntas relacionadas a la detección de autismo; potencialmente puede resultar una herramienta de tamizaje útil para estos propósitos. Estudios de validez predictiva podrían determinar su utilidad en esa dirección.

Las inferencias se limitan a la población de niños de la zona urbana donde se llevó a cabo el estudio y no necesariamente representar a otras zonas de la ciudad o regiones del país. No se mostraron valores de sensibilidad y especificidad de la prueba, debido a que no fueron objetivos del estudio. Sin embargo, pudiera resultar el inicio de estos estudios, teniendo en cuenta puntos de corte propios y actuales. 
Validación y puntos de corte de la versión en español del Cuestionario de Edades y Etapas: Social-emocional, 2da edición.

Como aplicación en la práctica clínica del $A S Q: S E-2$, además de ser un instrumento de ayuda en la detección de niños con riesgo de alteraciones sociales y emocionales, sirve de complemento a la aplicación del $A S Q-3$ en la identificación de niños con riesgo de trastornos del desarrollo psicomotor que requieren mayor evaluación.

Chen et al., en Brasil (16), en un estudio sobre la complementariedad del $A S Q-3$ con el $A S Q: S E-2$, realizado en 13470 niños de 1 a 4 años de edad, en escuelas públicas de Brasil con información reportada por padres y profesores. Mediante un análisis de correlación y regresión logística halló que las áreas de comunicación y personal-social según el $A S Q-3$ resultaron afectados y se reflejaban en la afectación socio-emocional según el $A S Q: S E-2$.

Aún son necesarios más estudios que determinen el valor predictivo de este tipo de pruebas de manera individual o juntas para establecer su utilidad como herramientas de detección del trastorno del espectro autista $(7,9-11)$.

En conclusión, el $A S Q: S E-2$ versión en español, como un instrumento con un constructo teórico del desarrollo socioemocional, completado por padres o cuidadores de niños entre los 15 meses 0 días y 53 meses 30 días de edad, en un entorno de consulta ambulatoria urbana de Lima, demostró ser válido y confiable para establecer riesgo de afectación de las habilidades socio-emocionales.

\section{Declaración de Financiamiento y de Conflictos de Intereses.}

Los autores declaramos no tener conflictos de interés, sean estos de orden económico, institucional, laboral o personal relevante para este artículo. Este estudio fue autofinanciado por los autores y no contiene una discusión de uso no aprobado/ investigativo de un producto/ dispositivo comercial.

Agradecimiento: Los autores agradecen a los padres de familia que participaron em el estudio. Al personal asistencial y administrativo de la Clìnica Mundo Salud, lugar donde se llevò a cabo el estudio y de manera especial a Oscar Ortiz A., por su apoyo en el procesamiento estadístico de los datos.

\section{Correspondencia:}

Adriel Gudiel Hermoza

Av. Carlos Izaguirre 1234, Los Olivos; código postal Lima-39, teléfono: 989011375

Correo electrónico: adrielgudielh@gmail.com

\section{REFERENCIAS BIBLIOGRÁFICAS}

1. Schore AN. Attachment, affect regulation, and the developing right brain: Linking developmental neuroscience to pediatrics. A review of the scientific foundations of current clinical practice. Pediatr Rev. 2005; 26(6): 204-217.

2. Council on Children with Disabilities, Section on Developmental Behavioral Pediatrics, Bright Futures Steering Committee, Medical Home Initiatives for Children with Special Needs Project Advisory Committee. Identifying Infants and Young Children with Developmental Disorders in the Medical Home: An Algorithm for Developmental Surveillance and Screening. Pediatrics. 2006; 118: 405-420.

3. Briggs R, Stettler E, Silver E, Schrag M, Chinitz S, Racine A. Social-emotional screening for infants and toddlers in primary care. Pediatrics. 2012; 129(2): 377-384.

4. Lipkin PH, Macias MM, Baer-Chen B, et al. Trends in pediatricians' developmental screening: 20022016. Pediatrics. 2020;145(4): e20190851.

5. Drotar D, Stancin T, Dworkin PH, Sices L, Wood S. Selecting developmental surveillance and screening tools. Pediatr Rev. 2008; 29 (10): e52-e58.

6. Marks KP, La-Rosa AC. Understanding developmental-behavioral screening measures. Pediatr Rev. 2012; 33 (10): 448-458.

7. Squires J, Bricker D, Twombly E. Ages \& Stages Questionnaires Social-Emotional second edition (ASQ-SE-2) User's Guide. Baltimore, MD: Paul H Brookes Publishing Co; 2015.

8. Squires J. Utilidad de padres y cuidadores como evaluadores certeros del desarrollo en niños menores. Rev Chil Pediatr. 2017; 88(1):22-24.

9. Squires J, Bricker D. Ages \& Stages Questionnaires: Third edition ASQ-3, in Spanish. Baltimore, MD. USA: Paul H. Brookes Publishing Co Inc.; 2009.

10. Lipkin PH, Macias MM.AAPCouncil on Children with Disabilities, Section on Developmental and Behavioral Pediatrics. Promoting optimal development: Identifying infants and young children with developmental disorders through developmental surveillance and screening. Pediatrics. 2020; 145(1): e20193449.

11. Dolata J.K. Early autism screening using a general social-emotional measure: Preliminary study of the ages and stages questionnaires: social emotional- $2 \mathrm{nd}$ 
edition. Tesis doctoral. Oregon: Graduate school of the University of Oregon; 2016.

12. Zumbo BD, Gadermann AM, Zeisser C. Ordinal versions of coefficients alpha and theta for likert rating scales. JMASM. 2007; 6 (1): 21-29.

13. Yovanoff P, Squires J. Determining cutoff scores on a developmental screening measure: Use of receiver operating characteristics and item response theory. J Early Interv. 2006; 29 (1):48-62.

14. Moodie S, Daneri P, Goldhagen S, Halle T, Green K, LaMonte L. Early childhood developmental screening: A compendium of measures for children ages birth to five (OPRE Report 2014 $\neg 11$ ). Washington DC: Office of Planning, Research and Evaluation, Administration for Children and Families, U.S. Department of Health and Human Services; 2014.
15. Heo KH, Squires J. Adaptation of a parent-completed social emotional screening instrument for young children: Ages and Stages Questionnaires-Social Emotional. Early Hum Dev. 2012;88(3): 151-158.

16. Chen CY, Anunciação L, Squires J, Filgueiras A, Landeira-Fernandez J. The relation between a developmental and social-emotional screening test used in public child daycare centers in Brazil. J Reattach Ther Dev Divers. 2019; 1(2):91-101.

Recibido: 14/08/2020 Aceptado: 02/06/2021 